\title{
Philosophiques
}

\section{Un conseil de Freud aux philosophes}

\section{Gérard Vachon}

Volume 16, numéro 1, printemps 1989

URI : https://id.erudit.org/iderudit/027063ar

DOI : https://doi.org/10.7202/027063ar

Aller au sommaire du numéro

\section{Éditeur(s)}

Société de philosophie du Québec

\section{ISSN}

0316-2923 (imprimé)

1492-1391 (numérique)

Découvrir la revue

\section{Citer cet article}

Vachon, G. (1989). Un conseil de Freud aux philosophes. Philosophiques, 16(1), 3-42. https://doi.org/10.7202/027063ar

\section{Résumé de l'article}

Dans son article de 1913, «L’intérêt de la psychanalyse», Freud déclarait que la philosophie pouvait profiter des lumières de la psychanalyse parce que celle-ci peut dévoiler la motivation subjective et individuelle de doctrines

philosophiques prétendument issues d'un travail logique et impartial, et ainsi désigner à la critique les points faibles du système d'un philosophe. Mon texte présente quelques exemples de l'utilisation de la psychanalyse pour identifier l'impact de l'inconscient sur la pensée de certains philosophes tels que Parménide, Berkeley, Kant, Sartre. À la lumière de ces exemples, j'examine brièvement quelques questions à propos du conseil de Freud : Est-il praticable ? Quand peut-on l'appliquer ? Comment en rendre l'application moralement acceptable ? Selon quels critères juger de la valeur de vérité d'interprétations psychanalytiques de ce genre ? Quels sont les bénéfices, et en valent-ils la peine ? 


\title{
AR'TICLES \\ UN CONSEIL DE FREUD AUX PHILOSOPHES
}

\author{
par Gérard Vachon
}

RÉSUMÉ. Dans son article de 1913, "L'intérêt de la psychanalyse", Freud déclarait que la philosophie pouvait profiter des lumières de la psychanalyse parce que celle-ci peut dévoiler la motivation subjective et individuelle de doctrines philosophiques prétendument issues d'un travail logique et impartial, et ainsi désigner à la critique les points faibles du système d'un philosophe. Mon texte présente quelques exemples de l'utilisation de la psychanalyse pour idencifier l'impact de l'inconscient sur la pensée de certains philosophes tels que Parménide, Berkeley, Kant, Sartre. À la lumière de ces exemples, j'examine brièvement quelques questions à propos du conseil de Freud: Est-il praticable? Quand peut-on l'appliquer? Comment en rendre l'application moralement acceptable? Selon quels critères juger de la valeur de vérité d'interprétations psychanalytiques de ce genre? Quels sont les bénéfices, et en valent-ils la peine?

ABSTRACT. In his article of 1913, « The Claims of Psycho-Analysis to Scientific Interest », Freud stated that philosophy could profit from psychoanalysis because it can indicate the subjective and individual motives of philosophical theories which are ostensibly based on impartial logical work, and in this way it can draw critical attention to the weak spots in a philosopher's system. This paper presents a few examples of the use of psychoanalysis to identify the impact of the unconscious on the theories of certain philosophers, e.g., Parmenides, Berkeley, Kant, Sartre. In the light of these examples, I examine briefly a number of questions concerning Freud's recommendation: Can it be put into practice? In what circumstances? How can it be applied in a way that is morally defensible? By what criteria can one evaluate the truth value of such psychoanalytic interpretations? What are the advantages, and are they worth the effort required? 


\section{INTRODUCTION}

En 1913, Freud publiait dans la prestigieuse revue Scientia un article intitulé « L'intérêt de la psychanalyse ». Pendant longtemps, ce texte était difficile d'accès en traduction française; nous devons à Paul-Laurent Assoun d'en avoir fourni, en 1980, non seulement une nouvelle traduction, mais aussi une préface et un commentaire qui font ressortir l'importance de cet article. ${ }^{1}$ Depuis, en 1984, la traduction du professeur Assoun est également parue dans la collection «Bibliothèque de psychanalyse», dirigée par Jean Laplanche. ${ }^{2}$

La revue Scientia offrait à Freud une excellente occasion de présenter la psychanalyse «à un cercle de savants qui s'intéressent à la synthèse des sciences ". ${ }^{3}$ Cette «jeune science» pouvait légitimement revendiquer l'intérêt d'autres sciences, y compris la philosophie, à laquelle Freud consacrait un court exposé intitulé "L'intérêt philosophique », dans lequel il offrait deux conseils aux philosophes.

En premier lieu, la philosophie doit tenir compte des contributions de la psychanalyse à la psychologie, comme elle le fait pour tous les progrès les plus importants des sciences spécialisées. En particulier, les philosophes doivent prendre connaissance des phénomènes de l'activité psychique inconsciente et éviter deux erreurs qu'ils ont - sauf quelques exceptions - entretenues : celle de faire de l'inconscient quelque chose de mystique, insaisissable et intangible dont la relation au psychique était dans l'obscurité, ou bien l'erreur d'identifier le psychique avec le conscient et de conclure que l'inconscient ne pouvait être l'objet de la psychologie.

Le deuxième conseil, celui que nous voulons étudier ici, était formulé ainsi :

La philosophie peut encore obtenir une stimulation de la psychanalyse d'une autre manière, c'est-à-dire dans la mesure où elle devient ellemême l'objet de celle-là même. Les doctrines et systèmes philosophiques sont l'œuvre d'un nombre réduit de personnes d'une frappe individuelle éminente; dans aucune autre science, la personnalité du travailleur

1. Sigmund Freud, L'intérêt de la psychanalyse, Paris, RETZ - C.E.P.L., 1980

2. Sigmund Freud, Résultats, idées, problèmes, l, 1890-1920, Paris, Presses Universitaires de France, 1984, pp. 187-213.

3. Ibid., p. 188. 
scientifique ne joue approximativement un si grand rôle, plus que précisément dans la philosophie. Or, ce n'est que la psychanalyse qui nous met en état de donner une psychographie de la personnalité... Ainsi, la psychanalyse peut dévoiler la motivation subjective et individuelle de doctrines philosophiques qui sont prétendument issues d'un travail logique impartial et désigner à la critique les points faibles du système. S'occuper de cette critique même n'est pas l'affaire de la psychanalyse, car, ainsi qu'on peut le concevoir, la détermination psychologique d'une doctrine ne concerne aucunement sa justesse scientifique. ${ }^{4}$

L'inconscient peut être un des facteurs déterminants de la pensée de tout homme, et donc le conseil de Freud pourrait s'appliquer à tous. Cependant, le rôle de l'individu étant encore plus important, en philosophie, que dans d'autres sciences, l'impact de l'inconscient sur la pensée du philosophe méritait, aux yeux de Freud, une attention particulière.

Dans ce qui suit, nous allons: (1) signaler des difficultés que présentent l'application du conseil de Freud ; (2) étudier quelques cas où l'on a tenté d'identifier l'influence de l'inconscient sur la pensée de certains philosophes; et (3) tirer quelques conclusions qui se dégageront de notre enquête.

\section{LES DIFFICULTÉS D'APPLICATION DU CONSEIL DE FREUD}

On trouve assez peu d'exemples de tentatives d'identifier l'effet de l'activité psychique inconsciente sur le travail des philosophes. Une première raison qui vient à l'esprit serait que, d'un côté comme de l'autre, les relations entre Freud et les philosophes ont, la plupart du temps, pris le caractère de la confrontation plutôt que celui de la collaboration. ${ }^{5}$

Le texte que nous venons de voir est relativement affable envers les philosophes. Freud leur adresse bien quelques reproches, mais les invite poliment à corriger leurs erreurs. Il reconnaît à la philosophie le titre de science, tout comme à la psychanalyse, et il parle en termes élogieux des qualités des philosophes. Finalement, il invite les philosophes à profiter des lumières de la psychanalyse,

4. libid., pp. 201-202.

5. Pour une étude très éclairante des relations de Freud avec les philosophes et $l_{a}$ philosophie, voir Paul-Laurent Assoun, Freud, la philosophie et les philosophes, Paris, P.U.F., 1976. 
mais souligne que c'est à la philosophie de faire ce travail et de juger elle-même de la justesse scientifique de ses doctrines.

Habituellement, Freud était loin d'être aussi courtois envers les philosophes. Par exemple, quelques années plus tôt, en 1909, il les combinait avec les psychologues pour leur assener le reproche suivant :

Il serait très désirable que les philosophes et les psychologues, qui élaborent par ouj-dire, ou à l'aide de définitions conventionnelles, d'ingénieuses doctrines sur l'inconscient, fissent d'abord des observations concluantes en étudiant les phénomènes de la pensée obsessionnelle ; on pourrait presque l'exiger, si cette tâche n'était de beaucoup plus pénible que leurs méthodes habituelles de travail. ${ }^{6}$

Ou encore, beaucoup plus tard, en 1932, dans les Nouvelles conférences, il déclarait:

La philosophie ne s'oppose pas à la science; se comportant elle-même comme une science, elle en emprunte aussi parfois les méthodes, mais s'en éloigne en se cramponnant à des chimères, en prétendant offrir un tableau cohérent et sans lacunes de l'univers, prétention dont tout nouveau progrès de la connaissance nous permet de constater l'inanité. Au point de vue de la méthode, la philosophie s'égare en surestimant la valeur cognitive de nos opérations logiques et en admettant la réalité d'autres sources de la connaissance, telle que par exemple, l'intuition. ?

Un peu plus loin, il ajoutait :

Plus encore, notre philosophie, est-ce niable? a conservé quelques traits essentiels du mode de penser animiste : la surestimation de la magie verbale, l'idée que notre pensée guide et régit les phénomènes réels. Il s'agit là, bien entendu, d'un animisme sans actes magiques. ${ }^{8}$

Ces citations donnent un aperçu du genre de problèmes que Freud voyait dans la pratique de la philosophie:

- la méconnaissance de l'inconscient;

- la prétention d'offrir un tableau complet de l'univers;

- la surestimation de la valeur cognitive de nos opérations logiques ;

6. Sigmund FreUd, “L'homme aux rats», Cinq psychanalyses, Paris, P.U.F., 1985, PP. 247-248.

7. Sigmund FREUD, Nouvelles conférences sur la psychanalyse, Paris, Gallimard, 1952, p. 219.

8. Ibid., p. 225. 
- l'acceptation d'autres sources de connaissances, telle l'intuition;

- la surestimation de la magie verbale, (l'omnipotence de la pensée).

Des déclarations de ce genre, et sur ce ton, n'étaient pas de nature à encourager de bonnes relations entre la philosophie et la psychanalyse. Par contre, l'hostilité de nombreux philosophes envers les théories freudiennes prenait souvent un ton tout aussi virulent que celui de Freud.

Sans doute, ce climat belliqueux rend compte en partie du fait que le conseil de Freud n'ait pas été appliqué très fréquemment par ceux à qui il était adressé. Cependant, il y a à cela d'autres raisons plus profondes: celui qui tente de suivre ce conseil se bute à de grandes difficultés.

Chacun peut essayer de déceler pour soi-même les influences inconscientes qui affectent sa propre pensée, mais l'autoanalyse ne peut pas mener très loin sur cette voie. Les philosophes pourraient subir une sorte de psychanalyse didactique afin de se sensibiliser aux effets de l'inconscient. Cependant, peu de philosophes se prêteraient à cette approche, et, de toute façon, cela ne les rendrait pas imperméables aux jeux de l'inconscient.

Qui, parmi les philosophes, pourrait se charger de révéler les influences inconscientes sur la pensée de ses collègues? Pour oser aborder ce sujet, ne faudrait-il pas être à la fois psychanalyste et philosophe ? Confier cette tâche à un organisme " officiel » porterait atteinte à la dignité et à la liberté des philosophes; une telle forme de dirigisme serait totalement inacceptable et dangereuse.

Il faut reconnaître également que les moyens utilisés en psychanalyse pour découvrir les ruses de l'inconscient sont, la plupart du temps, très peu utilisables pour «donner une psychographie de la personnalité » d'un philosophe. Comment peut-on distinguer les conclusions fondées sur des raisonnements solides, de celles qui ont été faussées par l'inconscient?

Finalement, en concentrant l'attention sur la psychologie d'un philosophe, on risque de négliger le contenu de son œuvre et de tomber dans l'argumentation ad bominem. 
Malgré ces difficultés, on peut trouver des tentatives pour identifier les effets de l'inconscient sur la pensée des philosophes: nous allons en étudier quelques exemples. Il faut souligner qu'aucun de ces exemples n'est rattaché explicitement au conseil de Freud formulé dans l'article, «L'intérêt de la psychanalyse ». Cependant, comme il s'agit bien de tentatives d'expliquer certains aspects de théories philosophiques à partir de notions psychanalytiques appliquées à leur auteur, nous sommes en droit de les considérer comme des illustrations de l'application du conseil de Freud.

\section{$1^{\text {er }}$ exemple: Jean-Paul Sartre}

Sartre a manifesté un intérêt constant pour la psychanalyse et a même proposé une psychanalyse existentielle en vue de remplacer celle de Freud. Quelques auteurs ont fait appel aux théories psychanalytiques pour expliquer certains éléments de sa philosophie, ce qui en fait un exemple particulièrement intéressant pour notre enquête. Finalement, il a lui-même expliqué de la même façon quelques aspects de la philosophie de Merleau-Ponty.

L'opposition de Sartre à la psychanalyse freudienne est exprimée à plusieurs reprises dans L'être et le néant, mais ce n'est que dans le dernier chapitre, intitulé « Faire et Avoir », qu'il expose son projet de la remplacer par la psychanalyse existentielle. Une de ses tâches sera de "dégager le sens ontologique des qualités », c'est-à-dire de tenter une psychanalyse des choses. "C'est ce que M. Bachelard a essayé avec beaucoup de talent dans son dernier livre, L'eau et les rêves. " ${ }^{9}$

Cependant, Sartre signale plusieurs points fondamentaux de désaccord avec l'approche de Bachelard, et c'est pour illustrer la méthode correcte qu'il présente sa propre psychanalyse de «cette qualité particulière qu'on nomme le visqueux.»10

Bachelard a répliqué à cette analyse dans la Terre et les rêveries de la volonté. Mais avant de le faire, il offre un commentaire très pertinent pour notre propos, en rapport avec le personnage de Roquentin de La nausée. Il dit:

9. Jean-Paul SARTRE, L'être et le néant, Paris, Gallimard, 1984, p. 661.
10. Ibid., p. 666. 
Ce personnage est désormais un type psychologique si bien défini qu'il faut le juger sans aucune référence à son créateur. Il a vraiment une vie propre. Nous souscrivons à ce propos à la remarque d'Emmanuel Mounier (Esprit, juillet 1946, p. 82) : «On voudra bien ne pas prendre cette remarque pour une psychanalyse existentielle de la pensée de Sartre : les liens ne sont pas nécessairement directs entre une personnalité et les idées qu'elle exprime ». Il est temps de libérer de ses créations le créateur. ${ }^{11}$

Cette citation de Mounier et le commentaire de Bachelard rejoignent une des difficultés que nous avons signalées ci-haut. Cependant, les deux philosophes ne parlent pas de la même chose. Bachelard parle d'un personnage créé par Sartre, tandis que Mounier parle de l'analyse que Sartre a faite de l'effet du regard d'autrui dans L'être et le néant. ${ }^{12}$

Il arrive sans doute très souvent qu'un personnage dans une œuvre littéraire exprime des vues diamétralement opposées à celles de l'auteur. Par contre, quand un philosophe expose ses opinions sur un sujet philosophique, on peut présumer qu'il présente ce qu'il pense. Il serait sans doute plus difficile de justifier l'utilisation des affirmations d'un personnage littéraire plutôt que des textes proprement philosophiques pour y trouver des traces de l'inconscient dans la pensée d'un philosophe qui, comme Sartre, a produit des ouvres dans les deux domaines. Mais, même ici, la différence n'est pas absolue, puisque Sartre s'est lui-même identifié à Roquentin, tout au moins pendant un certain temps. ${ }^{13}$

Enfin, notons que Mounier n'exclut pas la possibilité qu'il y ait des liens entre une personnalité et les idées qu'elle exprime. Il dit tout simplement que ces liens ne sont pas nécessairement directs. Cette observation ne l'empêche pas de signaler une ressemblance frappante entre les propos de Sartre et ceux du persécuté paranoïaque, ni d'affirmer :

On sait quelle valeur affective anormale prend dans son cuvre romancée comme dans sa réflexion le être-touché, fondement de la Nausée. Le

11. Gaston Bachelard, La terre et les rêveries de la volonté, Paris, Librairie José Corti, 1948 , p. 112, note 5.

12. Voir Emmanuel MoUnIER, Introduction aux existentialismes, Paris, Gallimard, 1962, FP. 120-123.

13. Voir Jean-Paul SarTre, Ler mots, Paris, Gallimard, 1975, p. 211. 
regard n'est que glaçant. Le monde des regards tend à installer la solitude glacée de l'univers paranoïaque. ${ }^{14}$

Mais revenons à Bachelard. Il semble bien avoir voulu s'abstenir de toute remarque qui aurait pu être interprétée comme s'appliquant à la psychologie de Sartre lui-même. Pourtant, l'analyse du visqueux de ce dernier fourmille de passages qui révèlent des influences inconscientes dans son raisonnement. En voici un cité par Bachelard:

c'est une activité molle, baveuse et féminine d'aspiration, il vit obscurément sous mes doigts et je sens comme un vertige, il m'attire en lui comme le fond d'un précipice pourrait m'attirer. Il y a comme une fascination tactile du visqueux. Je ne suis pas le maître d'arrêter le processus d'appropriation. Il continue. ${ }^{15}$

On peut se demander pourquoi une activité baveuse serait féminine, plutôt que masculine. Cependant, Bachelard évite toute référence à cette étrange manifestation de sexisme et de peur de la femme. Voici le début de son commentaire sur la citation que nous venons de voir.

Il continue sans doute si nous ne faisons rien, si nous vivons le visqueux dans son existence! Mais tout change si nous le travaillons. D'abord, dans le pétrissage, si la pâte colle aux doigts, une poignée de farine suffit pour nettoyer la main. Nous domestiquons le visqueux par l'artaque indirecte d'une matière sèche. Nous sommes démiurges devant le pétrin. Nous réglons le devenir des matières. ${ }^{16}$

Les associations que le visqueux inspire à Sartre sont tout à fait différentes, opposées même, à celles qui viennent à l'esprit de Bachelard. ${ }^{17}$ Cela pourrait bien sembler absolument sans conséquence, mais il faut tenir compte du fait que Sartre dégage le sens ontologique du visqueux à partir de ses associations.

Le visqueux apparaît comme un liquide vu dans un cauchemar et dont toutes les propriétés s'animeraient d'une sorte de vie et se retourneraient contre moi. Le visqueux, c'est la revanche de l'En-soi. Revanche

14. Emmanuel MOUNIER, Introduction aux existentialismes, p. 122.

15. Jean-Paul SARTRE, L'être et le néant, p. 671.

16. Gaston BACHELARD, La terre et les rêveries de la volonté, P. 116.

17. Voir les commentaires très pertinents d'Hélène Védrine sur les différences fondamentales entre les vues du monde des deux philosophes, dans son article au titre tellement suggestif : C Comment mettre Roquentin au travail... ou Bachelard et Sartre sur l'imaginaire», Gaston Bachelard. Profils épistémologiques, éd. par Guy Lafrance, Ottawa, Presses de l'Université d'Ottawa, 1987, pp. 109-119. 
douceâtre et féminine qui se symbolisera sur un autre plan par la qualité de sucré. ${ }^{18}$

Ici encore, le sexisme et la peur de la femme sont franchement exprimés, mais accompagnés d'un langage intellectualiste: «Le visqueux, c'est la revanche de l'En-soi. " Un peu plus haut, Sartre avait expliqué que la réalité humaine est choix d'être, soit directement, soit par appropriation du monde. Lorsque ce choix porte sur l'appropriation, chaque chose est choisie par suite de la manière dont elle rend l'être.

Revenons au projet originel. Il est projet d'appropriation. Il contraint donc le visqueux à révéler son être ; le surgissement du pour-soi à l'être étant appropriatif, le visqueux perçu est "visqueux à posséder », c'està-dire que le lien originel de moi au visqueux est que je projette d'être fondement de son être, en tant qu'il est moi-même idéalement... c'est précisément dans le cadre de ce projet appropriatif que le visqueux se révèle et développe sa viscosité. Cette viscosité est donc déjà - dès l'apparition première du visqueux — réponse à une demande, déjà don de so $i$; le visqueux paraît comme déjà l'ébauche d'une fusion du monde avec moi ; et ce qu'il m'apprend de lui, son caractère de ventouse qui m'aspire, c'est déjà une réplique à une interrogation concrète ; il répond avec son être même, avec sa manière d'être, avec toute sa marière. ${ }^{19}$

D'un côté, cette réponse est claire : le visqueux est ce dont je manque. D'un autre côté, la réponse est opaque: le visqueux renvoie à une signification pleine et dense et cette signification nous livre l'être-en-soi, en tant que le visqueux est présentement ce qui manifeste le monde. Tant que dure le contact avec le visqueux, tout se passe comme si la viscosité était le sens du monde tout entier, l'unique mode d'être de l'être-en-soi.

L'analyse du visqueux est suivie de l'analyse du trou.

Or, en lui-même, le trou est le symbole d'un mode d'être que la psychanalyse existentielle se doit d'éclaircir. ${ }^{20}$

Sartre déclare qu'il ne peut «y insister ici », mais il en dit assez pour que l'on puisse saisir où il veut en venir. Le trou se présente comme un néant à combler avec sa propre chair : l'enfant ne peut s'empêcher d'y mettre son doigt ou son bras entier. Boucher le trou

18. Jean-Paul Sartre, L'être et le néant, p. 671.

19. Ibid., pp. 667-668.

20. Ibid., p. 675 
c'est originellement sacrifier son corps pour que la plénitude d'être existe, c'est-à-dire subir la passion du Pour-soi pour façonner, parfaire et sauver la totalité de l'En-soi. L'enfant cherche la densité, la plénitude uniforme et sphérique de l'être parménidien.

Dans la suite des explications, on retrouve encore les deux niveaux du langage. Le message de la peur de la femme est encore énoncé de façon dramatique et combiné avec le message intellectualiste :

L'obscénité du sexe féminin est celle de toute chose béante: c'est un appel d'être, comme d'ailleurs tous les trous; en soi la femme appelle une chair étrangère qui doive la transformer en plénitude d'être par pénétration er dilution... Sans aucun doute le sexe est bouche et bouche vorace qui avale le pénis - ce qui peut bien amener l'idée de castration : l'acte amoureux est castration de l'homme - mais c'est avant tout que le sexe est trou.... c'est avec sa chair que l'enfant bouche le trou, et le trou, avant toute spécification sexuelle, est une attente obscène, un appel de chair. ${ }^{21}$

Sartre se pose des questions d'une franchise désarmante à propos de lui-même :

Si le visqueux est bien le symbole d'un être où le pour-soi est bu par l'ensoi, que suis-je donc moi qui, à l'encontre des autres, aime le visqueux ? À quel projet fondamental de moi-même suis-je renvoyé si je veux expliciter cet amour d'un en-soi enlisant et louche? 22

Sartre n'a pas répondu directement à ces questions, dans L'être et le néant. Le Dr Karl Stern fournit des éléments de réponse dans son ouvrage intitulé Refus de la femme. Essai. Le livre est, en partie, une application du conseil de Freud aux philosophes, puisqu'il révèle comment des motivations inconscientes ont influencé la pensée, non seulement de Sartre, mais aussi de Descartes, de Schopenhauer et de Kierkegaard.

On pourrait peut-être objecter que le conseil de Freud s'adressait aux philosophes, et qu'ici c'est un psychanalyste qui en fait l'application. Il serait dans l'ordre de répliquer que l'Essai du $\mathrm{Dr}$ Stern est tout autant le travail d'un philosophe que celui d'un psychanalyste. En plus d'apporter une contribution originale et

21. Ibid., p. 676 .

22. Ibid. 
profonde à la compréhension de la doctrice des philosophes déjà mentionnés, son livre contient de nombreux commentaires perspicaces et éclairants à propos de Merleau-Ponty, Husserl, Marx, Bergson, Simone de Beauvoir, Karl Jaspers, Dilthey et Bertrand Russell, pour ne nommer que ceux-là. De toute façon, même si les philosophes refusaient d'accueillir le Dr Stern parmi eux, ils auraient grand intérêt à considérer attentivement le matériel qu'il apporte. Mais voyons ce qu'il dit à propos de l'œuvre de Sartre.

Il signale, dans La nausée, dans l'étude sur Baudelaire, et dans Les mouches, des passages qu'il considère comme des exemples de ce que Freud appelle le processus primaire.

Ce n'est qu'en partant du processus primaire décrit par Freud que nous comprenons le thème directeur de la nausée dans le roman de Sartre, la raison pour laquelle des sentiments comme le vertige, le malaise, l'angoisse se mêlent aux spéculations métaphysiques de Roquentin. Car le monde du processus primaire, dans lequel l'homme est entouré d'images nues, non modifiées par l'abstraction, est normalement le monde de l'enfance... Faim, colère, joie, satiété, nausée, tristesse composent les éléments psychiques de ce que l'enfant accepte ou rejette. L'acceptation ou le refus de cẹt ordre constitue à cet âge le seul mode de relation avec autrui. Si l'on veut traduire ce qui précède en langage philosophique, supposons que Roquentin dise à Descartes : il ne sert à rien de tenir à distance la res extensa. On est inextricablement mêlé à la matière, dans un amas gluant, sale, visqueux, qui fait vomir. ${ }^{23}$

Quand Roquentin décrit sa réaction au monde sous la forme de l'expérience orale primitive, il traduit une intuition profonde. Il ne faut pas croire que, parce qu'il s'agit d'un ouvrage littéraire, cela n'aurait rien à voir avec la partie philosophique de l'œuvre de Sartre, et notamment L'être et le néant.

La nausée renferme une profusion de mots qui connotent quelque sorte d'Urmaterie, de matière primitive d'expériences orales et tactiles (par exemple visqueux, gluant, suintant, grouillant, se tortillant, bourgeonnant, etc.), un déploiement de qualités primitives, que nous retrouvons comme de vieilles connaissances, mais plus dispersées, dans l'œuvre théorique de Sartre. ${ }^{24}$

Dans l'univers de Sartre, le pour-soi rejette l'en-soi comme on se défait d'un amas gluant. Lorsqu'on y rencontre la sexualité,

23. Karl Stern, Refus de la femme. Essai, Montréal, Éditions HMH, 1968, p. 115.

24. lbid., p. 118. 
elle est froidement physiologique ou répugnante. On n'y trouve aucune belle figure de femme. Le conflit est le mode fondamental de tout rapport humain : c'est un système de possession où l'amour est irréalisable.

Ce n'est qu'aujourd'hui, à l'époque de Sartre, que la séparation d'avec la matière et le maternel atteint à l'absolu. ${ }^{25}$

L'analyse du visqueux que nous avons suivie en partie dans L'être et le néant est sans doute un autre exemple du processus primaire qui pourrait être ajouté à ceux présentés par le Dr Stern. On y voit, clairement exposées dans le même texte, à la fois les réactions primitives qui provoquent l'horreur du visqueux, et leur conversion en une métaphysique.

En 1970, un philosophe et un médecin américains, Ben-Ami Scharfstein et Mortimer Ostow, dans un article intitulé «The Need to Philosophize», cirèrent une partie du texte que nous avons vu plus haut dans lequel Sartre décrit la femme comme celle qui appelle une chair étrangère (Note 21). Immédiatement après cette citation, vient le commentaire suivant :

Sartre believes that the threat of « the hole " is presexual. But if we pay attention not to this abstract idea but to the tone of the passage, we must find it both cruel and pained, as his writing often is... Sartre's philosophy is constructed on sadomasochistic lines. The chief participants in Being and Nothingness are, as the name indicates, Being, which is full, imperturbable, and godlike, and Nothing, or human consciousness, which cannot exist without Being, yet which must prey on it. Consciousness is Nothing because it is a constant grasping, a yearning and a failure to become Being. In Sartre's world, man is free, but only in the consciousness of his lack, and therefore in anguish. Love, too, therefore, is sadomasochistic and doomed. One tries to enslave the Other's freedom, but succeeds only in appropriating the Other's body. Love is necessary and yet futile. ${ }^{26}$

Cette appréciation des idées de Sartre est très semblable à celle qu'en faisait le Dr Stern, mais souligne en plus la tendance sadique-masochiste.

25. Ibid., p. 121.

26. Ben-Ami Scharfstein et Mortimer Ostow, "The Need to Philosophize ", Psychoanalysis and Philosophy, éd. par Charles Hanly et Morris Lazerowitz, New York, International Universities Press, 1970, pp. 270-271. 
On peut se demander si ces deux auteurs ainsi que le Dr Stern ont eu tort de faire, pour ainsi dire, une «psychanalyse » de Sartre, dont se sont abstenus Mounier et Bachelard. À leur appui, on doit reconnaître que leurs conclusions sont fondées sur les textes du philosophe lui-même.

Sartre a fait appel lui aussi à des notions psychanalytiques pour expliquer certains éléments de la philosophie de MerleauPonty. Voici quelques passages tirés de l'article qu'il consacrait à ce dernier dans le numéro spécial de la revue Les temps modernes, en octobre 1961.

Et puis, il souffrait de ses rapports avec autrui : tout avait été trop beau trop vite ; la Nature qui l'enveloppa d'abord, ce fut la Déesse Mère, sa mère, dont les yeux lui donnaient à voir ce qu'il voyait; elle fut l'alter ego; par elle, en elle, il vécut cette « intersubjectivité d'immanence » qu'il a souvent décrite et qui nous fait découvrir par l'autre notre "Spontanéité ». ${ }^{27}$

Plus loin, à propos de l'impact sur Merleau-Ponty de la mort de sa mère, Sartre écrit :

On se répète sans cesse, on ne recommence jamais. En voyant sombrer son enfance il se comprit: il n'avait jamais rien souhaité que la rejoindre et cet impossible désir était sa vocation singulière, sa destinée... Renvoyé pour la troisième fois à lui-même par cette mort, il voulut éclairer par elle sa naissance. Au nouveau-né, à ce voyant-visible qui paraît dans le monde de la vision, quelque chose doit advenir, n'importe quoi, ne fût-ce que mourir. Cette tension première entre l'apparition et la disparition, il la nomme «historicité primordiale » : c'est en elle et par elle que tout arrive; elle nous précipite du premier instant dans une irréversibilité inflexible. ${ }^{28}$

Ces deux citations montrent bien que Sartre ne répugnait aucunement à rattacher la pensée d'un philosophe à sa vie psychique, même s'il rejetait en grande partie la psychanalyse freudienne, surtout l'existence de l'inconscient.

27. Jean-Paul SARTrE, Situations IV, Gallimard, 1964, p. 197.

28. Ibid., p. 263 et $265-266$. 


\section{$2^{e}$ exemple : Le livre de John Oulton W isdom sur Berkeley}

J.O. Wisdom est un philosophe qui connaît bien la psychanalyse et qui a consacré beaucoup d'attention aux liens entre les deux disciplines. Avant de publier son livre sur Berkeley, il avait déjà étudié l'impact de l'inconscient dans l'œuvre de Schopenhauer ${ }^{29}$ et sur certains aspects de la philosophie de Descartes. ${ }^{30} \mathrm{Comme} \mathrm{dans}$ le cas de son article sur Schopenhauer, le titre de son volume sur Berkeley, The Unconscious Origin of Berkeley's Philosopby, ${ }^{31}$ indique clairement l'objectif poursuivi, mais il s'agit ici d'un travail beaucoup plus élaboré.

La première partie examine les théories philosophiques de Berkeley à la lumière des recherches les plus récentes disponibles au moment de la rédaction. Cette analyse doctrinale était nécessaire à cause des nombreuses opinions divergentes qui circulaient sur l'interprétation de l'œuvre de Berkeley, indépendamment de toute considération psychanalytique. La deuxième partie résume les faits connus à propos de la vie de Berkeley. La troisième partie présente et explique 56 « interprétations » qui affirment des liens entre les théories de Berkeley et sa vie psychique, en utilisant la documentation assemblée dans les deux premières parties. Lorsque Wisdom avance des interprétations qu'il ne peut pas appuyer sur cette documentation, il le signale clairement.

Dans son chapitre final, l'auteur énumère quelques critères qui aideraient à juger de la valeur des explications de ce genre. En voici un résumé :

1. Les interprétations doivent être cohérentes entre elles.

2. Elles ne doivent pas contredire aucun fait connu à propos du sujet.

3. L'analyse devrait éclairer un problème (The analysis should throw light on some problem). Wisdom souligne qu'en général les interprétations psychanalytiques ne décrivent

29. J.O. WISDOM, "The Unconscious Origin of Schopenhaner's Philosophy », The International Journal of Psycho-Analysis, 1945, Vol. XXVI, pp. 44-52.

30. J.O. WISDOM, "Three Dreams of Descartes», The International Journal of PsychoAnalysis, 1947, Vol. XXVIII, pp. 11-18.

31. J.O. WISDOM, The Unconscious Origin of Berkeley's Philosophy, London, The Hogarth Press and the Institute of Psycho-Analysis, 1953. 
pas des causes dont les effets peuvent être prédits de façon précise; elles décrivent plutôt, la plupart du temps, les causes d'un effet déjà connu.

4. Les interprétations doivent être du même type que celles faites dans la clinique, et préférablement utilisées largement dans la pratique clinique.

5. L'épreuve la plus importante serait l'effet des interprétations sur un patient, mais ceci manque entièrement dans des interprétations comme celles dont nous parlons. ${ }^{32}$

Malgré la qualité du travail de Wisdom, il faut dire que certaines de ses interprétations laissent le lecteur mal à l'aise, même celui qui connaît les théories freudiennes et ne les rejette pas automatiquement. Dans plusieurs cas, ses interprétations reposent sur des conclusions établies par l'expérience clinique de nombreux psychanalystes, comme l'exige son quatrième critère, mais sans confirmation dans les faits connus à propos de Berkeley.

Avant d'exposer un exemple de cette lacune, il faut voir une partie du parcours préalable de Wisdom. Après avoir démontré comment Berkeley souffrait d'une peur exagérée du poison, et après avoir établi des liens entre cette peur et sa doctrine selon laquelle la matière n'existait pas, ainsi qu'avec sa foi absolue dans les vertus curatives de l'eau de gaudron, Wisdom tente d'expliquer l'ambivalence de Berkeley envers la matière. D'un côté, elle n'existait pas, mais il fallait néanmoins déployer des efforts énormes pour l'éliminer sous la forme du poison. Wisdom offre l'interprétation que Berkeley souffrait d'un sentiment de culpabilité inconscient à cause d'un désir refoulé d'avoir quelque chose d'intimement relié au poison. Jusqu'ici, Wisdom fournit des justifications, quelquefois ténues, mais dont le lecteur peut prendre note et évaluer la valeur démonstrative.

Cependant, à la prochaine étape, Wisdom déclare qu'il faut aller plus loin et trouver le précurseur de tous ces genres de poison, c'est-à-dire la matière fécale. Il avance donc l'interprétation selon laquelle, pour Berkeley, à l'âge adulte les fèces étaient du poison. ${ }^{33}$ Comme Wisdom le souligne, les liens psychiques entre le poison et

32. Voir ibid., pp. 227-229.

33. Voir ibid., les interprétations VIII et IX, et leur présentation. 
la matière fécale ont été souvent identifiés en clinique. Mais, le fait que l'adulte Berkeley ait été fortement préoccupé par le poison, et le fait que plusieurs psychanalystes aient souvent confirmé, dans la clinique, un lien entre poison et matière fécale, ne justifient pas la conclusion que, pour l'adulte Berkeley, les fèces étaient du poison. Il faudrait en plus connaître quelques faits à propos de l'enfance de Berkeley qui confirmeraient que chez lui il y a bien eu une évolution semblable à celle que les psychanalystes ont établie dans de nombreux cas.

On retrouve le même problème tout au long du chapitre 20 intitulé «Childhood Reconstructions » où Wisdom avance d'autres interprétations sur les fèces, les relations avec les pères (divin et humain), les premières relations objectales, la présence et l'absence, et finalement, le temps. La plupart de ces interprétations sont conformes à des expériences cliniques bien établies parmi les psychanalystes, mais sans aucun appui dans les faits connus à propos de l'enfance de Berkeley. Ceci ne veut pas dire que ces interprétations soient nécessairement fausses; elles sont toutes plausibles comme hypothèses.

Cependant, dans un domaine aussi controversé, il conviendrait d'ajouter un autre critère à ceux énumérés par le professeur Wisdom, c'est-à-dire que, pour être acceptable, une interprétation qui propose un lien entre l'adulte et l'enfant devrait s'appuyer sur des éléments connus à propos de la vie adulte et l'enfance du sujet.

Les hypothèses plus spéculatives pourraient être conservées, mais nettement séparées des interprétations qui satisfont à tous les critères, y compris celui que nous proposons. Elles devraient en plus porter un autre nom : peut-être celui de «constructions». En effet, les hypothèses dont nous parlons sont très semblables à celles que Freud décrivait dans son article de 1937, «Constructions dans l'analyse $»$. Il disait :

... on peut parler de construction quand on présente à l'analysé une période oubliée de sa préhistoire... Nous n'attribuons à la construction isolée que la valeur d'une supposition qui attend examen, confirmation ou rejet. ${ }^{34}$

34. Sigmund Freud, «Constructions dans l'analyse», Résultats, idées, problèmes, $I I$, 1921-1938, Paris, P.U.F., 1987, Pp. 273 et 277. 
Le mot «reconstructions », dans le titre du Chapitre 20, va déjà dans le sens que nous proposons, d'autant plus que Freud considérait les deux mots comme équivalents: "Son travail de construction ou, si l'on préfère, de reconstruction... » ${ }^{35}$ Cependant, les «interprétations » moins bien justifiées, y compris celles contenues dans le chapitre 20 sont toutes identifiées et numérotées de la même façon que les interprétations plus solidement documentées, ce qui pourrait laisser l'impression qu'elles sont toutes mises sur un pied d'égalité. La crédibilité de l'ensemble de l'analyse serait augmentée en gardant le nom «interprétation» pour les conclusions bien documentées, et en les séparant des conclusions plus hypothétiques, qui, elles, porteraient un autre nom, tel que «construction» ou « reconstruction».

Avant de passer à notre prochain exemple, il est utile de situer brièvement la démarche de Wisdom. Dans un autre livre publié beaucoup plus récemment, en 1975, Philosophy and Its Place in Our Culture, il explique que lorsqu'il commença son enquête sur l'origine inconsciente de la philosophie, le positivisme logique était à son apogée, et prétendait que le métaphysique était dénuée de signification. Wisdom se demandait alors comment autant de grands penseurs avaient pu comprendre tant d'affirmations dépourvues de sens et les discuter rationnellement pendant si longtemps. Il se rendit compte que la métaphysique serait dénuée de signification seulement si on acceptait la position positiviste selon laquelle une proposition doit se rapporter à l'expérience perceptive pour être porteuse de sens. Les philosophes, en répliquant aux positivistes, auraient insisté sur la signification transcendentale de la métaphysique.

Positivists had at least, however inadvertently, drawn attention to the difficulty of grasping or articulating transcendental meaning. And I came to the conclusion that it might refer to a transcendental world in the sense of a world picture as a projection of a philosopher's own unconscious structure. ${ }^{36}$

35. Ibid., p. 271.

36. J.O. WISDOM, Philosophy and lts Place in Our Culture, New York, London, Paris, Gordon and Breach Science Publishers, 1975, p. 190. 
Wisdom, contrairement aux positivistes, n'avait pas comme objectif l'élimination de la métaphysique. Il répète plusieurs fois sa conviction que même si l'on démontre qu'une thèse philosophique a ses origines dans des motifs inconscients, ceci ne constitue aucunement une réfutation de la thèse. ${ }^{37}$

\section{$3^{\mathrm{e}}$ exemple : Les travaux de Morris Lazerowitz}

Morris Lazerowitz a lui aussi beaucoup écrit à propos des motivations inconscientes qui influent sur la pensée des philosophes. Son point de départ était semblable à celui de Wisdom. ${ }^{38} \mathrm{Il}$ se demandait comment il était possible de débattre les mêmes problèmes philosophiques pendant des millénaires sans jamais arriver, selon lui, à aucun accord, sans réaliser aucun progrès. ${ }^{39}$

En vue de découvrir la nature de la métaphysique, il examine tour à tour quatre hypothèses et les rejette toutes comme étant inadéquates.

1. Les théories métaphysiques ne sont pas de nature empirique ; elles ne s'appliquent pas aux phénomènes.

2. Elles ne sont pas des propositions a priori. Lazerowitz rejette, comme Wisdom, la théorie kantienne selon laquelle une proposition synthétique a priori peut exprimer quelque chose à propos des phénomènes. ${ }^{40}$

3. Les théories métaphysiques ne sont pas des affirmations portant sur l'usage correct du langage.

4. Elles ne sont pas dénuées de signification. Ici l'auteur s'attaque, comme Wisdom encore une fois, au positivisme logique.

Ces quatre hypothèses ayant été rejetées, Lazerowitz en propose une cinquième selon laquelle les théories métaphysiques

37. Wisdom donne son appréciation, très critique, de l'analyse philosophique, du positivisme logique et de la philosophie linguistique, ibid., pp. 40-44.

38. Wisdom compare ses propres vues et celles de Lazerowitz, ibid., PP. 193-195.

39. Le professeur Lazerowitz a présenté ses thèses à de nombreuses reprises. Ici, nous en résumons certains aspects surtout à partir du chapitre intitulé "The Nature of Metaphysics ", The Structure of Metaphysics, London, Routledge and Kegan Paul, 1955, pp. 23-79; et de l'article "The Relevance of Psychoanalysis to Philosophy", Psycboanalysis, Scientific Metbod and Philosopby, éd. par Sidney Hook, New York, New York University Press, 1959, pp. 133-156.

40. Voir The Structure of Metaphysics, pp. 261-268. 
seraient des innovations linguistiques déguisées. Ainsi, Iorsqu'un philosophe dit: "Le changement n'existe pas", il s'exprime comme s'il déclarait quelque chose à propos des phénomènes. Cependant, il utilise cette expression comme si elle était une proposition a priori, du même genre que : «Toutes les tulipes sont des fleurs». La seule façon dont la phrase: «Le changement n'existe pas » peut être utilisée comme une proposition a priori, c'est en modifiant l'usage établi : c'est-à-dire en enlevant au mot "changement » son application aux choses matérielles. À l'inverse, le philosophe qui prétend que «tout est changement continuel » élargit, sans le dire, l'extension du mot changement, de sorte que l'expression " ne pas être en changement " n'a plus d'application aux choses matérielles.

Jusqu'à ce stade, la théorisation de Lazerowitz ne s'appuie aucunement sur la psychanalyse. Il s'agit d'une argumentation purement logique et linguistique. Son approche s'inspire beaucoup de Wittgenstein : en parlant de sa propre théorie sur la nature de la philosophie, il déclare que c'est «an account which is merely a development of Wittgenstein's thought. » ${ }^{41}$

W ittgenstein aurait traversé une période iconoclaste de courte durée après son retour à Cambridge en 1929. C'est pendant cette période qu'il dicta The Blue and Brown Books qui contiennent plusieurs des réflexions qui ont inspiré Lazerowitz. En parlant de ces réflexions, ce dernier écrit :

Their implication is that a philosopher changes language in one way or another under the illusion that he is expressing a proposition about what there is (or is not) and about the nature of what there is. The further implication is that the revised piece of language is semantically idle, i.e., that it has no actual use to communicate information, yet creates the illusion of expressing a speculation about the world. ${ }^{42}$

Mais, pour Lazerowitz, Wittgenstein semble avoir préféré exprimer ses perceptions dans un langage métaphorique plutôt que direct, peut-être en partie pour atténuer l'aspect troublant de son message.

41. M. Lazerowitz, The Language of Philosophy. Freud and Wittgenstein, Dordrecht et Boston, D. Reidel Publishing Company, Boston Studies in the Philosophy of Science, Vol. LV, 1977, p. 29

42. Ibid., p. 30. 
Remarks like " philosophical problems arise when a language goes on holiday" and philosophical language is "like an engine idling, not when it is doing work » give expression to disturbing perceptions into the nature of technical philosophy but use a form of the mechanism of sotto voce to deflect them. Where their translations into prosaic language would tend to stir up anxiety, these words can be accepted as colorful jibes which need not be taken seriously. ${ }^{43}$

Si Wittgenstein a mis une sourdine à ses affirmations iconoclastes, Lazerowitz a proclamé les siennes hautement et sans équivoque. En restant toujours dans son argumentation fondée sur la logique et la linguistique, nous pouvons noter certaines opinions auxquelles il a donné un caractère absolu, e.g.,

- en 2,400 ans la philosophie n'a pas réussi à produire une seule proposition qui ne soit pas encore un sujet de controverse. ${ }^{44}$

- une proposition a priori ne peut contenir aucun renseignement sur le monde. ${ }^{45}$

- toutes les parties de la philosophie, y compris l'éthique, l'esthétique, l'épistémologie, ainsi que la métaphysique, souffrent du même problème. ${ }^{46}$

De telles affirmations ont naturellement suscité des positions contraires. Cependant, la théorisation de Lazerowitz qui a provoqué le plus de protestations est celle que nous allons voir maintenant, celle où il fait appel à la psychanalyse.

La conclusion selon laquelle les théories métaphysiques ne seraient que des innovations linguistiques déguisées soulève la question de savoir pourquoi les philosophes, pour la plupart, ne se sont pas rendu compte depuis longtemps de cet état de choses, et pourquoi ils continuent leurs débats interminables sur quelque chose qui semble tellement banal. C'est pour expliquer cet étrange phénomène que Lazerowitz introduit la psychanalyse dans sa théorisation.

43. Ibid., p. 43.

44. Voir M. Lazerowitz, Philosophy and Illusion, London, George Allan \& Unwin Ltd., et New York, Humanities Press, 1968, p. 82.

45. Voir M. LAZEROWITZ, The Language of Philosophy. Freud and Wittgenstein, p. 185.

46. Voir M. LAzerowitz, Studies in Metaphilosophy, London, Routledge and Kegan Paul, 1964, p. ix. 
Une théorie métaphysique comporterait trois niveaux :

1. Au niveau conscient, elle crée l'illusion qu'elle s'applique au monde des phénomènes.

2. La proposition qui contient la théorie change l'utilisation normale d'un ou plusieurs mots, sans que l'auteur s'en rende compte.

3. La proposition semble exprimer une théorie, mais en réalité elle exprime des croyances (beliefs) de l'inconscient. ${ }^{47}$

Nous allons maintenant retracer brièvement les nombreuses présentations que Lazerowitz a faites des théories que nous venons de voir. Ensuite, nous tenterons de faire une évaluation de la partie de ses travaux qui peut être considérée comme constituant des exemples de l'application du conseil de Freud.

Le premier livre de Lazerowitz qui expose ces théories est paru en 1955, The Structure of Metaphysics. Il reproduisait plusieurs articles déjà publiés entre 1939 et 1952, ainsi que quelques nouveaux textes. En 1958, avait lieu à New York la deuxième réunion annuelle du New York University Institute of Philosophy, dont une partie des délibérations est parue en 1959, sous le titre Psychoanalysis, Scientific Method and Philosophy. Le professeur Lazerowitz fut le premier conférencier de la partie du programme portant sur la psychanalyse et la philosophie. Son texte résumait la théorisation déjà présentée dans The Structure of Metaphysics.

La réplique des professeurs Donald $\mathrm{C}$. Williams et Antony Flew, comme la discussion subséquente, fut presqu'entièrement négative et fondée en bonne partie sur l'émotion plutôt que sur des considérations rationnelles. Par contre, le professeur John Hospers apporta son appui à une partie des théories de Lazerowitz. En évoquant la violence de la réaction contre celles-ci, il se demanda si les philosophes s'attaquaient aux principes freudiens eux-mêmes ou à la tentative de Lazerowitz d'appliquer ces principes à certains philosophes qu'il avait choisis comme exemples. Le dernier texte du compre-rendu, celui du professeur Campbell Crockett, porte le

47. Voir, e.g., The Structure of Metaphysics, pp. 67-79; Psychoanalysis, Scientific Method and Philosophy, p. 136 et p. 153; Philosophy and Illusion, PP. 109-110. 
titre révélateur : «Misunderstanding One Another ». Son auteur, sans être d'accord avec la thèse principale de Lazerowitz, déplore la superficialité des critiques hostiles provoquées par sa présentation. ${ }^{48}$

Ces réactions négatives n'empêchèrent pas Lazerowitz de continuer l'exposition de ses vues, en 1964, dans Studies in Metaphilosophy, et en 1968, dans Pbilosophy and Illusion. Dans la préface de ce dernier livre, il explique brièvement le programme qu'il s'était tracé. L'objectif d'une partie de ce programme était de construire une hypothèse qui expliquerait à la fois le manque de résultats stables en philosophie, et le fait que cette discipline continuait toujours de susciter un grand intérêt. La deuxième partie du programme visait à appliquer l'hypothèse à des problèmes fondamentaux et représentatifs dans les diverses branches de la philosophie : la métaphysique, l'épistémologie, l'éthique, l'esthétique, la philosophie des sciences, etc. Il déclarait que ce programme était, d'une certaine façon, complété, même s'il restait encore beaucoup à faire. ${ }^{49}$ Cependant, on retrouve les mêmes thèses dans un autre ouvrage publié en 1977: The Language of Pbilosophy. Freud and Wittgenstein.

Dans les divers articles des quatre livres que nous avons énumérés, les thèses que nous avons déjà résumées sont inlassablement appliquées à une grande variété de sujets tels que l'existence des universaux, le mouvement, l'espace, le temps, la perception, l'induction, et beaucoup d'autres. Comme chaque article est plus ou moins autosuffisant, on trouve beaucoup de répétitions d'un article à l'autre, mais aussi des nuances nouvelles ou de nouvelles façons d'exprimer les mêmes idées.

Comme nous l'avons signalé plus haut, la partie de l'argumentation qui est fondée sur la logique et la linguistique est très élaborée. Par contre, les exemples donnés pour illustrer la motivation inconsciente des théories de divers philosophes sont très peu

48. Le texte de la présentation de Lazerowitz est intitulé «The Relevance of Psychoanalysis to Philosophy »et se trouve dans Psychoanalysis, Scientific Method and Pbilosopby, pp. 133-156; les répliques des professeurs Williams et Flew, aux pp. 157-197; le texte du professeur Hospers aux pp. 336-357, et celui du professeur Crockett aux pages $360-363$.

49. Voir Philosopby and Illusion, p. 13. 
documentés. On peut constater clairement cette lacune dans la présentation faite au symposium de 1958 à New York.

Lorsque vient le moment d'introduire l'influence de l'inconscient pour expliquer la permanence et l'intensité de l'intérêt pour les questions philosophiques, Lazerowitz signale qu'un des messages inconscients que les théories philosophiques expriment semblerait rattaché à une curiosité infantile insatisfaite. Il cite ensuite quelques passages tirés de l'Introduction à la psychanalyse dans lesquels Freud explique que l'intérêt sexuel de l'enfant se porte en premier lieu sur le problème de savoir d'où viennent les enfants; que l'enfant n'est pas dupe des explications folkloriques (e.g. la cigogne) que lui donnent les adultes; et que l'impression d'avoir été trompé contribue à l'isolement de l'enfant et au développemment de son indépendance; mais que l'enfant n'est pas à même de résoudre ce problème par ses propres moyens.

Lazerowitz affirme ensuite, sans plus de justification, que la description que Freud donne du caractère d'une personne dont la curiosité infantile n'a pas été satisfaite convient parfaitement à Spinoza. Il ne serait donc pas surprenant que Spinoza ait cherché inconsciemment une réponse à sa curiosité infantile. Ses propres paroles justifieraient une telle interprétation lorsqu'il dit "que toutes choses sont en Dieu et dépendent de lui de telle sorte que, sans lui, elles ne peuvent ni être, ni être conçues ». ${ }^{50}$ Lazerowitz souligne les mots «ne peuvent ni être, ni être conçues» et déclare que ces paroles exprimeraient, presque sans déguisement, une théorie à propos de l'état de dépendance de l'enfant et à propos de la procréation. On ne serait pas surpris d'apprendre que le mot "cause " soit utlisé inconsciemment pour symboliser le père ou Dieu (la première cause). En affirmant que quelque chose ne peut pas être produit par rien, Spinoza révélerait son inquiétude à l'égard du problème de la naissance et son incapacité de le résoudre. En disant que sans Dieu les choses ne pourraient pas exister ni être conçues, il exprimerait une vague perception selon laquelle son propre père aurait joué un rôle dans sa création, mais il n'aurait jamais pu satisfaire son désir inconscient de connaître ce qu'avait été ce rôle. Ceci serait démontré par la toute première définition de L'Éthique: "Par cause de soi, j'entends ce dont

50. SPINOZA, L'İthique, Paris, Gallimard, 1964, p. 62. 
l'essence enveloppe l'existence, autrement dit ce dont la nature ne peut être conçue qu'existante ». ${ }^{51}$ Cette définition indiquerait que la question de savoir comment son père était venu au monde était demeurée un mystère pour lui, et par conséquent comment luimême était venu au monde serait également resté un mystère. La déclaration philosophique, «Il ne peut y avoir un événement sans une cause " exprimerait une proposition qui apaisa un désir infantile de connaissance, mais sans le satisfaire. ${ }^{52}$

Aucun fait de la vie de Spinoza n'est invoqué à l'appui de ces conclusions. On pourrait penser que les contraintes de temps et d'espace, lors du symposium de 1958, auraient peut-être été la cause de ce manque de documentation. Cependant, dans un autre article, entièrement consacré à Spinoza, la justification n'est guère plus élaborée. Lazerowitz utilise un fait de la vie de Spinoza, son excommunication de la communauté juive, pour expliquer un aspect de la théorie de Spinoza, mais il admet lui-même que son explication n'est qu'une conjecture (a guess). ${ }^{53}$

En examinant les autres articles des quatre livres de Lazerowitz déjà cités, on constate partout la même faiblesse, lorsqu'il s'agit de faire le lien entre la théorie freudienne et la vie ou les écrits du philosophe auquel des motivations inconscientes sont attribuées. Ces attributions ne sont pas nécessairement fausses ; cependant, sans documentation, sans au moins quelques éléments de preuve, elles ne peuvent réclamer aucune crédibilité, surtout devant des lecteurs sceptiques ou carrément hostiles.

Nous avons noté plus haut quelques déclarations tranchées que l'on trouve dans les textes de Lazerowitz. La même tendance se manifeste dans la partie de ses travaux où il fait appel à la psychanalyse, e.g.,

— une «théorie» philosophique n'est pas une théorie, et un argument philosophique n'est pas une démonstration ni une réfutation $;{ }^{54}$

51. Ibid., p. 19

52. Voir Psychoanalysis, Scientific Method and Pbilosophy, pp. 152-153.

53. Voir The Language of Philosophy, p. 139.

54. Voir Studies in Metaphilosophy, p. 217. 
- le philosophe, sans le savoir, utilise le langage uniquement pour exprimer des pensées inconscientes; ${ }^{55}$

- le philosophe, comme le poète, obtient le succès et le respect du monde intellectuel au moyen d'un rêve inventé qu'il peut partager avec d'autres; 56

- le philosophe est un jongleur qui joue de vains tours avec la grammaire. ${ }^{57}$

Dans cette veine, il faut noter que Lazerowitz se veut délibérément plus radical que Freud. En effet, il déclare qu'à sa connaissance aucun psychanalyste, y compris Freud, n'est parvenu à l'idée, ou même au soupçon de l'idée que le métaphysicien ne recherche pas la vérité, comme il le croit lui-même et comme le croient d'autres personnes. Il reproche au Dr Michael Balint d'avoir affirmé que c'était à la métaphysique de répondre à la question de savoir laquelle de deux images du monde est la bonne. En parlant de l'ouvrage de J.O. Wisdom sur Berkeley, il déclare n'y avoir trouvé que la notion classique à propos de la nature de la philosophie, c'est-à-dire, la notion que les théories philosophiques sont des déclarations portant sur l'existence et la nature des phénomènes. ${ }^{58}$

Lazerowitz ne cite pas le passage dans «L'intérêt de la psychanalyse » où Freud déclare que c'est la philosophie qui doit faire la critique de ses propres théories, car «la détermination psychologique d'une doctrine ne concerne aucunement sa justesse scientifique ». Il n'aurait sans doute pas été d'accord avec cette affirmation de Freud, puisqu'il reproche à Balint d'avoir énoncé essentiellement la même thèse.

Les quatre recueils d'articles dont nous avons parlé ont reçu, en général, le même accueil hostile que la présentation de 1958 à New York. Les faiblesses que nous venons de voir motiveraient ces critiques en partie, sans toutefois justifier la hargne de quelquesuns de leurs auteurs. ${ }^{59}$

55. Voir Philorophy and Illurion, p. 80.

56. Voir The Language of Philosophy, p. 25.

57. Voir ibid., p. 199

58. Voir Psychoanalysis, Scientific Method and Pbilosopby, p. 135

59. Voici quelques exemples des recensions des quatre livres:

- D.D. RAPHAËL, "The Structure of Metaphysics. By Morris Lazerowitz», The Philosophical Quarterly, Vol. 7, 1957, pp. 80-86.

- K. Daya, “Some Considerations on Morris Lazerowitz's" "The Structure of Metaphysics», Mind, Vol. LXVIII, 1958, pp. 236-243. 
Même s'ils ne sont pas bien documentés, les liens que Lazerowitz suggère entre les théories de nombreux philosophes et leur inconscient, pourraient très bien être valables. Dans un de ses articles, "The Nature of Metaphysics », il exprime la conviction que tôt ou tard la philosophie deviendra un domaine auquel les psychanalystes consacreront plus d'attention. Il déclare également :

Certainly philosophy is of sufficient importance as an intellectual phenomenon to deserve to have more light thrown on it than it has had up to the present, and by investigators who do not have a vested interest in it. ${ }^{60}$

Dans cette perspective, les suggestions de Lazerowitz pourraient et mériteraient d'être approfondies et évaluées à la lumière d'études plus détaillées du genre de celles que J.O. Wisdom a faites.

Signalons un autre recueil d'articles, édité par Charles Hanly et Morris Lazerowitz, publié en 1970 et portant le titre Psychoanalysis and Philosophy. Nous allons nous arrêter brièvement sur deux de ces articles qui ont le mérite de fournir une solide documentation afin d'établir des liens entre les théories philosophiques et la vie psychique des philosophes qui les ont élaborées.

\section{4e exemple : L’article de Charles Hanly sur Parménide}

L'article de Charles Hanly est intitulé «On Being and Dreaming ". "II Il étudie l'ouvre de Parménide afin de vérifier une hypothèse seion laquelle des théories métaphysiques seraient composées de propositions hybrides qui combineraient la syntaxe logique des énoncés scientifiques (sans être, comme ceux-ci, vérifiables) et les significations anthropomorphiques de la poésie. ${ }^{62}$

- J.W.N. WatKINS, "Word Magic and the Trivialization of Philosophy", Ratio, Vol. 7, 1965, pp. 206-218

- Brand Blansharo, "Morris Lazerowitz. Philosophy and Illusion ", Metaphilosophy, Vol. 2, 1970, pp. 178-185.

- Susan HaAck, «Morris Lazerowitz. The Language of Philosophy. Freud and Wittgenstein ", Metaphilosophy, Vol. 10, 1979, pp. 340-342.

60. The Structure of Metaphysics, p. 67.

61. Charles Hanı.Y, "On Being and Dreaming", Psychoanalysis and Philosopby, éd. par C. Hanly et M. Lazerowitz, New York, International Universities Press, 1970, pp. $155-187$.

62. Voir ibid., p. 160. 
Le début du poème de Parménide (De la nature) serait un rêve contenant un oracle. Hanly montre que de tels rêves étaient pris au sérieux dans la Grèce antique et qu'ils étaient interprétés, contrairement à la pratique psychanalytique, d'après leur contenu manifeste plutôt que d'après leur contenu latent.

Parménide aurait intégré le contenu de son rêve dans les méditations que le rêve aurait suscité chez lui. Donc, le professeur Hanly voit le poème entier comme un tout, profondément unifié, et il considère l'introduction comme étant essentielle à la compréhension de l'ensemble.

In essence, what the goddess told Parmenides was that the name of the world is being. Although trivial to us because of our more realistic grasp of the nature of language, such a « discovery " was by no means trivial to a prescientific Greek. Given the magical identification of the name and the named, to have the name of the Real is to be placed in the position of being able to enter into an ontological, moral and cognitive identification with the Real;... The goddess enjoined Parmenides to think and say of Reality only that « it is » and never that « it is and is not $»$. Reality is sheer static positivity and identity. ${ }^{63}$

Le professeur Hanly fait ressortir de nombreux autres liens entre l'introduction du poème et la doctrine exposée dans « La voie de la vérité » et dans « La voie de l'opinion ». Il utilise abondamment les théories psychanalytiques pour interpréter le poème, en indiquant toujours son raisonnement et ses sources.

Nous voulons surtout concentrer notre attention sur la fin de l'article, où Hanly examine la question de la valeur de vérité d'une interprétation psychologique comme celle qu'il vient de donner. En parlant de l'article de Freud, «L'intérêt de la psychanalyse », il écrit :

Freud (1913) adopted the position that the question as to whether or not a religious idea serves as a wish fulfillment psychologically is logically distinct from and irrelevant to the question of its truth. ${ }^{64}$

Il s'agit du passage que nous avons cité dans lequel Freud parle de la critique des théories philosophiques (non pas religieuses), et où il déclare que « la détermination psychologique d'une doctrine ne concerne aucunement sa justesse scientifique. ${ }^{65}$

63. Ibid., p. 170.

64. Ibid., p. 185.

65. Voir, ci-haur, la citarion portant le $n^{\circ} 4$. 
Le professeur Hanly continue :

That they are logically distinct questions is obvious, but that they are always independent is not so obvious.

Cette formulation pourrait donner l'impression que Freud lui-même aurait affirmé que les deux questions [(1) qu'une idée serve ou non à satisfaire un désir; (2) la valeur de vérité de cette idée] sont toujours indépendantes, ce que Freud n'a pas dit. Mais là n'est pas l'essentiel de notre propos.

Hanly distingue ensuite deux sortes de relations entre une représentation consciente, intellectuelle, et un fantasme inconscient auquel la première est associée. Cette relation peut être soit contingente, soit constitutive. Lorsque la relation est constitutive, le fantasme détermine le contenu et l'utilisation des idées conscientes. Le refus systématique de toute vérification empirique serait un signal qu'une relation constitutive existe. Une explication et une interprétation psychologiques seraient appropriées lorsqu'il s'agit d'idées qui sont à la fois vagues et protégées contre la falsification par une épistémologie qui répudie la vérification par l'observation. Nous reviendrons à ce critère; il est sans doute très utile pour identifier les points faibles que Freud recommandait de soumettre à la critique.

La toute dernière phrase de l'article se lit comme suit: "Metaphysics is visionary poetry in the logical syntax of mathematics and natural science. " ${ }^{66}$ Hanly semblerait ainsi appliquer son hypothèse du début à toute la métaphysique. Cependant, on se demande comment une application aussi générale pourrait être justifiée, puisque l'auteur n'a présenté qu'un cas qui se prêtait à la confirmation de son hypothèse. Il se peut cependant que, malgré les apparences, le professeur Hanly n'ait voulu appliquer cette phrase qu'au cas de Parménide.

\section{$5^{\mathrm{e}}$ exemple : L'article de Lewis S. Feuer sur Kant}

Avant de publier l'article que nous allons étudier, le professeur Feuer avait en 1957 publié, sur un thème semblable, un autre excellent article intitulé «The Dream of Benedict de Spinoza ». ${ }^{67}$

66. Ibid., p. 186.

67. Lewis S. Feuer, "The Dream of Benedict de Spinoza», The American Imago, Vol. XIV, 1957, pp. 225-242. 
En 1958, lors du symposium de New York, il avait, comme Lazerowitz, subi les foudres du professeur Donald C. Williams, qui déclarait :

To brim the cup of fantasy, Professor Lewis Feuer in a rival psychoanalysis of Spinoza has meanwhile produced such arbitrarily variant findings as that Spinoza, usually bracketed with Socrates as one of the two most self-reliant men in history, was a cringing masochist, so far from boasting of being God that he bleated to be a slave. ${ }^{68}$

Il suffit de lire l'article de Feuer pour constater la fausseté et l'injustice de ce commentaire de Williams ; c'est un bon exemple des réactions émotives et hargneuses mentionnées plus haut.

Mais passons à l'article paru dans le recueil édité par Hanly et Lazerowitz. Son titre indique clairement son objectif : «Lawless Sensations and Categorial Defenses : the Unconscious Sources of Kant's Philosophy ». ${ }^{69}$ Un des mérites de cet article est de montrer jusqu'à quel point la légende a déformé l'image de Kant en le dépeignant comme un être sans passion. Et c'est surtout Kant luimême qui a décrit ses propres conflits, ses appréhensions, ses dégoûts, ses tendances irrationnelles. Cependant, on trouve ces confidences dans des parties de son œuvre que l'on a l'habitude de considérer comme ayant peu ou pas de rapport avec sa philosophie.

Le professeur Feuer postule que chaque philosophe possède une "protophilosophie », c'est-à-dire une série d'axiomes qu'il ne met pas en doute. Ceux-ci reflètent l'attitude du philosophe envers la réalité avec ses emphases, conflits, refoulements et exagérations ; ces présuppositions expriment le point de vue émotif du philosophe. L'auteur soumet la "protophilosophie» de Kant à une étude psychanalytique, afin d'identifier les causes émotives, non-logiques, les plus profondes qui ont déterminé sa philosophie.

Dans cette étude, il part de la prémisse selon laquelle $L a$ critique de la raison pure est fondée sur deux propositions : (1) que nous ne connaissons pas les choses en soi, mais seulement la façon selon laquelle elles nous apparaissent en tant que phénomènes; et

68. Donald C. WILliams, Psychoanalysis, Scientific Method and Philosophy, p. 177.

69. Lewis S. Feuer, "Lawless Sensations and Categorial Defenses: the Unconscious Sources of Kant's Philosophy", Psychoanalysis and Philosophy, Pp. 76-125. 
(2) que nos fonctions mentales imposent sur nos sensations des formes et des structures nécessaires et universelles.

Feuer donne à la première de ces propositions le titre de «estrangement assumption»; ${ }^{70}$ c'est-à-dire que les sensations ne sont pas fiables, quelles constituent un mur entre nous et la réalité. Il décrit ensuite le genre de personne qui est enclin, pour des raisons émotives, à déprécier le rôle des sensations. De telles personnes cherchent à refouler ou à retenir leur sexualité au moyen de divers mécanismes de défense. Surtout, ce sont des personnes pour qui ce qu'elles reçoivent des sens évoque un sentiment de culpabilité.

L'odorat inspirait un grand dégoût à Kant. Freud a noté que l'hostilité envers l'odorat était caractéristique des personnes qui sont offensées par la sexualité. Kant a également exprimé son malaise à propos de la vue. Il déclarait que beaucoup d'hommes sont incapables de faire abstraction d'un défaut qu'ils voient chez des femmes qu'ils pourraient autrement aimer. Kant était grandement distrait si un bouton manquait au manteau d'un de ses étudiants, ce qui aurait évoqué le déshabillage, et provoqué la crainte de perdre le contrôle de ses sentations. Kant est devenu aveugle temporairement à deux reprises, et le professeur Feuer est d'avis qu'il s'agissait d'une cécité "which was evidently neurotic in its origin. $)^{71}$

Kant se sentait obligé de résister à la soif. Il ne voulait pas être vu lorsqu'il buvait du thé. Les sens étaient une autre source de perturbation pour lui. Il les comparait aux odeurs qui se répandent dans toutes les directions et que l'on doit subir même contre son gré.

Feuer apporte beaucoup d'autres indications, toujours documentées, pour appuyer sa thèse selon laquelle Kant souffrait d'une grande méfiance émotive envers toutes les formes de sensation. Il passe ensuite au deuxième postulat qu'il voit dans la "protophilosophie» de Kant - celui selon lequel la raison impose à notre expérience des formes et des catégories nécessaires. Il entend démontrer que la déduction transcendantale des catégories

70. 1 bid., p. 77

71. Ibid., p. 80 
était l'expression camouflée d'éléments psychologiques hautement personnalisés.

And as a neutoric, intent on preserving order, Kant imposed on the lawless data his a priori categories of the understanding. The «transcendental deduction » projected his own introspective experience of controlling what orherwise would be a subversive world. ${ }^{72}$

L'auteur déclare que la méthode adoptée par un philosophe est son postulat le plus important. Dans le cas de Kant, ce qui est le plus important à propos de la méthode transcendantale, c'est ce qu'elle n'est pas. Malgré les affirmations contraires de Kant, la déduction transcendantale est une application voilée de la méthode psychologique. Les catégories permettent d'éviter la folie. Leur "déduction" est fondée sur l'argument selon lequel nous serions comme des aliénés mentaux si nous ne les acceptions pas. Il s'agit d'une étude de la psychologie de la santé mentale. ${ }^{73}$

Quelle est donc cette «déduction transcendantale 》 qui est empirique sans l'être, qui discute des conditions qui rendent possible l'expérience rationnelle humaine, mais qui est censée n'avoir rien à voir avec les lois causales de la psychologie ? 74

Le professeur Feuer retrace ce qu'il appelle «The Antipsychological Revolution in Kant's Life ». Pendant sa jeunesse Kant était un enthousiaste de la méthode psychologique. Mais, au milieu de sa vie, il se tourna contre cette méthode, ce qui l'obligea à faire la distinction entre le moi transcendantal et le moi empirique.

The transcendental self never fitted well into Kant's system. In fact, it was one of those " points of inconsistency » which cast a light on the philosopher's underlying conflicts. It was solely a formal presupposition ; you knew the transcendental «I » existed, but you knew nothing more about it. To say that such a noumenal ego existed, without knowing some identifying characteristics about it, contravened Kant's own restriction of knowledge to the phenomenal realm. ${ }^{75}$

Dans ce passage, Feuer fait allusion à un critère qu'il avait énoncé auparavant dans son article, et qui, comme celui donné par Hanly, permet d'identifier des cas où la doctrine d'un philosophe est peut-être influencée par son inconscient.

72. lbid., p. 84

73. Ibid., p. 105

74. Ibid., p. 108

75. Ibid., p. 110 
In studying the psychoanalytic basis of a philosophy, a most important indicator is found in the points of inconsistency in the given philosophy, i.e., a place where the philosopher's underlying emotion is constrained by his own doctrine... The point of inconsistency is a place where a quantum of the philosopher's raw emotion has not been rationalized into philosophical form. ${ }^{76}$

Dans de telles circonstances, le besoin de l'inconscient triompherait sur la logique du philosophe et l'obligerait d'adopter, à son insu, une position en contradiction avec les autres éléments de sa doctrine. Feuer est d'avis que la résistance de Kant envers la théorie de l'évolution était un tel point de contradiction (a point of inconsistency) tout comme sa distinction entre le moi trancendantal et le moi empirique, et il consacre une bonne partie de son article à la démonstration des effets de l'inconscient sur la pensée de Kant en rapport avec ces deux points.

\section{UN BILAN PROVISOIRE}

À la lumière des exemples que nous avons étudiés, nous allons tenter de répondre aux six questions suivantes :

a) Est-il techniquement possible d'appliquer le conseil que Freud donnait aux philosophes?

b) Si la réponse est affirmative, dans quelles circonstances est-il à propos de le mettre en pratique?

c) Quelles règles devraient être suivies afin que l'application soit moralement acceptable?

d) Selon quels critères peut-on juger de la valeur de vérité des interprétations psychanalytiques en rapport avec les théories d'un philosophe?

e) Quels sont les bénéfices qu'on peut retirer d'une telle entreprise?

f) Le jeu en vaut-il la chandelle?

\section{A) Un conseil techniquement praticable?}

Parmi les exemples que nous avons examinés, le cas de Sartre est celui dont certains éléments de théorisation semblent le mieux se prêter à une interprétation psychanalytique. On pourrait aller 76. Ibid., p. 95-96. 
plus loin, et dire qu'une telle interprétation est nécessaire, afin de comprendre quelques-unes de ses théories les plus fondamentales. Si on s'arrête au message intellectualiste, on ne pénètre pas vraiment dans les sources profondes de la pensée de Sartre. Par exemple, comme le faisait remarquer le Dr Stern, ce n'est qu'en partant du processus primaire décrit par Freud que l'on peut comprendre le thème de la nausée, que l'on trouve dans l'œuvre littéraire et à la base de certains aspects de l'œuvre théorique de Sartre.

Peu de philosophes se sont confiés aussi franchement que Sartre. Nous avons vu cependant que Kant a révélé beaucoup de lui-même dans des parties de son œuvre que l'on a tendance à séparer totalement de sa philosophie. Feuer, en utilisant ce matériel et des biographies de Kant, a identifié des liens qu'il considère comme déterminants entre la vie psychique du philosophe et ses théories.

J.O. Wisdom disposait de peu de documentation sur la vie de Schopenhauer, mais il a trouvé dans Le monde comme volonté et comme représentation de nombreuses affirmations dans lesquelles il a pu discerner des influences inconscientes. Dans le cas de Berkeley, beaucoup d'éléments de sa vie adulte étaient connus, mais peu de son enfance, ce qui empêchait la vérification de certaines des hypothèses avancées par J.O. Wisdom. Ses autres interprétations étaient, par contre, bien documentées, de sorte que le lecteur peut évaluer leur valeur démonstrative.

Ces exemples confirment qu'il est techniquement possible d'appliquer le conseil de Freud aux philosophes, mais ils montrent également que le degré d'applicabilité varie énormément, selon la nature et l'étendue des renseignements disponibles.

\section{B) Quand I'appliquer?}

Deux de nos auteurs ont chacun fourni un critère pour découvrir des cas où la pensée d'un philosophe a peut-être été faussée par l'inconscient. Le premier est celui de Hanly d'après lequel c'est un signe d'une telle situation lorsqu'un philosophe présente une idée vague et la protège contre la falsification au moyen d'une épistémologie qui rend l'observation impossible, e.g., l'idée de Parménide que l'Être doit être sphérique. 
Le deuxième critère est celui de Feuer : lorsqu'un élément est en contradiction avec d'autres éléments de la doctrine d'un philosophe, il se peut qu'un désir inconscient ait obligé le penseur, à son insu, de satisfaire ce besoin. On pourrait répliquer qu'il n'est pas nécessaire de faire appel à la psychanalyse pour déceler des contradictions. C'est vrai, mais il ne s'agit pas nécessairement, ici, de contradictions flagrantes. Il s'agit au contraire de failles finement camouflées, e.g., les objections de Kant contre la théorie de l'évolution, et sa distinction entre le moi transcendantal et le moi empirique. Le critère de Feuer encourage le lecteur à regarder de plus près des points où le raisonnement est difficile à suivre et où on se demande si l'argumentation est compatible avec d'autres aspects de la théorie.

Il reste, cependant, que le simple fait de savoir ou de soupçonner que l'inconscient influence la pensée peut aiguiser le sens critique et ainsi aider à déceler des défauts de logique qui pourraient autrement passer inaperçus. Lorsqu'une telle lacune a été ainsi constatée, il n'est pas toujours nécessaire ni même utile de «psychanalyser » le philosophe en question. Prenons deux exemples bien connus.

On peut penser qu'Adam Smith a succombé un peu vite à la tentation de trouver dans la libre entreprise une clef magique qui servirait au mieux à la fois les intérêts des producteurs et des consommateurs. L'examen critique de la base de sa théorie montre qu'un marché totalement libre n'a jamais existé et qu'il est impossible de le réaliser, du moins sur une grande échelle.

De la même façon on peut penser que Marx s'est laissé emporter par un désir semblable lorsqu'il a proclamé que la dictature du prolétariat se transformerait en société sans classe. Un peu de réflexion critique montre l'invraisemblance d'une telle évolution, et la faiblesse des arguments présentés.

\section{C) Comment en rendre l'application moralement acceptable?}

Il est difficile d'être plus désagréable et plus injuste que le psychanalyste amateur qui se permet de juger sommairement tous ceux qu'il rencontre en leur infligeant une psychanalyse instantanée. 
Ce genre de comportement est totalement inacceptable moralement, et Freud aurait été le premier à s'élever contre de tels abus.

Mais quelles règles de conduite peuvent rendre légitime l'application du conseil de Freud ? On peut trouver des éléments de réponse dans la déontologie médicale et légale ainsi que dans la pratique des historiens. Le secret professionnel protège la vie privée du client ou du patient. Les archivistes s'abstiennent d'utiliser des documents avant la date spécifiée par leurs donateurs. D'une manière analogue, celui qui a des raisons de croire que tel ou tel aspect d'une doctrine philosophique a été développé sous l'influence de l'inconscient doit s'abstenir d'utiliser des renseignements de caractère privé à propos d'un philosophe vivant, à moins que ce dernier ne les ait révélés lui-même publiquement ou qu'il ait permis leur utilisation. Les documents confiés par un philosophe à des archives doivent évidemment être traités selon les mêmes règles que tout autre legs.

Il faut également exercer un contrôle strict afin de s'assurer que les renseignements utilisés soient véridiques. Finalement, celui qui tente de mettre en pratique le conseil de Freud doit à ses lecteurs ou auditeurs d'identifier avec soin ses sources de renseignements ainsi que le raisonnement qui appuie ses conclusions.

\section{D) La valeur de vérité des interprétations}

Il va sans dire que beaucoup de gens, y compris beaucoup de philosophes, n'acceptent aucunement les théories de la psychanalyse, et par conséquent ne peuvent trouver aucune valeur dans les tentatives d'identifier les forces inconscientes qui peuvent avoir influencé la pensée d'un philosophe.

Cependant, lorsque ces tentatives révèlent des faiblesses dans les démonstrations d'un philosophe, et que ces lacunes sont exposées par les techniques de la logique ou par d'autres moyens, même ceux qui sont sceptiques ou hostiles envers la psychanalyse pourront tirer profit de cette argumentation. Ils n'ont pas le droit de rejeter ces démonstrations simplement parce qu'elles auraient été précédées d'une analyse utilisant des notions psychanalytiques.

Ceux qui acceptent les théories psychanalytiques peuvent utiliser les critères que J.O. Wisdom a proposés, en leur ajoutant 
quelques autres règles que nous avons identifiées ci-haut. On peut résumer ces critères ainsi :

1. Les interprétations doivent être cohérentes entre elles.

2. Elles doivent être conformes aux faits connus à propos du philosophe.

3. Elle doivent éclairer un problème qui se présente dans la compréhension de la doctrine d'un philosophe, e.g., l'ambivalence de Berkeley à propos de la matière.

4. Les interprétations doivent être du même type que celles qui ont été souvent confirmées par les psychanalystes, e.g., formations substitutives; formations réactionnelles.

5. Une interprétation qui propose un lien entre l'adulte et l'enfant doit s'appuyer sur des faits connus à propos de la vie adulte et l'enfance du sujet, afin de démontrer que l'évolution affirmée a bien eu lieu.

6. Des hypothèses plausibles peuvent être présentées, mais elles doivent être identifiées comme telles et nettement séparées des interprétations.

\section{E) Les bénéfices}

Ici, nous allons, une fois de plus, nous inspirer en grande partie des observations de J.O. Wisdom. Dans son livre, Philosophy and Its Place in Our Culture, publié en 1975, donc 30 ans après son article sur Schopenhauer, et 22 ans après son livre sur Berkeley, il nous présente, à plusieurs reprises, divers résultats de sa longue méditation sur les facteurs inconscients qui peuvent influencer la philosophie.

Dans son chapitre intitulé «The Place of Goals and Motives in Philosophy », il se pose la question suivante:

What light may be thrown upon a philosophy by investigating its unconscious origin ? 77

Les bénéfices que Wisdom identifie sont très modestes. Premièrement, en étudiant l'origine inconsciente d'une philosophie, nous apprenons quelque chose que nous ne pourrions pas apprendre

77. Philosophy and lts Place in Our Culture, p. 191. 
autrement, à propos du philosophe. Mais, cette connaissance additionnelle augmente-t-elle notre compréhension de sa philosophie? Wisdom affirme qu'il est facile de voir qu'une perspective mieux équilibrée peut être obtenue.

... I think we fail to grasp the fulness of Leibnitz's philosophy if we do not understand it as the philosophy of a man dismayed by vanities and evils, and constantly haunted by certain horrors. Leibnitz - and also Spinoza - was saying that the universe is « all of a piece ", and that we are all, as elements reflecting or in causal connection with the rest, equally responsible for what goes on in it, and that we have to accept our position in it. This is in Leibnitz's philosophy, but is not easily discernible without the unconscious being investigated; and I think it considerably alters our understanding even of the content of the philosophy. ${ }^{78}$

Wisdom souligne que ce bénéfice se rattache à la compréhension (understanding) davantage qu'à la vérité ou la fausseté. Mais il touche également à cette dernière question. Ici, Wisdom rappelle ce qu'il a souvent dénoncé : «the genetic fallacy ». C'est-à-dire que selon lui, retracer l'origine d'une opinion n'en constitue pas une réfutation. Cependant, il faut prendre garde de ne pas exagérer:

too much awe of it can lead us to overlook something so that the respect for the genetic fallacy can lead to a one-sided assessment. For a knowledge of preconscious and unconscious motives can legitimately undermine a philosophy in the sense of leading us to suspect it, to suspect it of saying nothing about the world, a suspicion we may then look into further to see whether it is objectively undecidable - although, and here we again pay heed to the genetic fallacy, the undermining fails if we find an objective function in the philosophy. And investigations into unconscious structures can point to whether the structure is inherently pathological or oriented maturely towards a real conflict in real life. ${ }^{79}$

Donc, trois bénéfices peuvent être retirés de l'étude de l'origine inconsciente de la pensée d'un philosophe:

1) Une meilleure compréhension du philosophe et de sa philosophie ;

2) cette étude peut éveiller des doutes qui provoquent un retour critique sur la philosophie en question;

78. lbid., p. 192.

79. Ibid. 
3) ce retour critique peut conduire soit à la conclusion que l'origine inconsciente des idées du philosophe n'a pas affaibli la valeur objective de ses idées ni des preuves qu'il apporte, soit à la conclusion que l'origine inconsciente des idées et de l'argumentation a ruiné leur crédibilité, en partie ou totalement.

Il est frappant que ce que Wisdom identifie comme bénéfices provenant de l'étude des motivations inconscientes est tout a fait conforme à ce que Freud anticipait :

Ainsi, la psychanalyse peut dévoiler la motivation subjective et individuelle de doctrines philosophiques qui son prétendument issus d'un travail logique impartial et désigner à la critique des points faibles du système. ${ }^{80}$

\section{F) Le jeu en vaut-il la chandelle?}

Ici encore, la réponse sera radicalement différente pour ceux qui rejettent les théories freudiennes et pour ceux qui les acceptent. Les premiers considéreront comme entièrement futile toute tentative de trouver des influences inconscientes sous-jacentes aux théories philosophiques. Pour l'autre camp, il n'y a pas lieu de réclamer des avantages très éclatants, mais la plupart des exemples que nous avons étudiés permettent d'y voir, ni plus, ni moins, que les bénéfices promis par Freud et confirmés par Wisdom.

Si les enquêtes sur les influences inconscientes mènent à une meilleure compréhension de certains philosophes, à une critique plus rigoureuse de leurs œuvres, et à une confirmation ou à une invalidation de la valeur de leurs théories, en voilà assez pour justifier l'effort requis. Même ceux qui n'y croient pas pourront, s'ils ne refusent pas de le faire, profiter de cette critique plus exigeante occasionnée par une appréciation des effets possibles de l'inconscient.

En terminant, revenons à une question posée au tout début : qui va se charger de mettre en pratique le conseil de Freud? Il semble bien que ce seront, comme par le passé, quelques rares

80. Voir ci-haut la ciration portant le $n^{0} 4$ 
philosophes qui, pour une raison ou une autre (peut-être inconsciemment), s'intéressent personnellement à ces questions.

\section{Université d'Ottawa}

\section{BIBLIOGRAPHIE}

ASSOUN, Paul-Laurent, Freud, la philosophie et les philosophes, Paris, P.U.F., 1976.

BACHELARD, Gaston, La terre et les rêveries de la volonté, Paris, Librairie José Curti, 1948.

FEUER, Lewis S., "The Dream of Benedict de Spinoza ", The American Imago, Vol. XIV, 1957, pp. 225-242.

"Lawless Sensarions and Categorial Defenses : the Unconscious Sources of Kant's Philosophy », Psychoanalysis and Pbilosopby, éd. par C. Hanly et M. Lazerowitz, New York, International Universities Press, 1970, Pp. 76-125.

FREUD, Sigmund, (1909), "L'homme aux rats", Cinq psychanalyses, Paris, P.U.F., 1985, Pp. 199-261.

, (1913) "L'intérêt de la psychanalyse ", Résultats, idées, problèmes $I$, 1890-1920, Paris, P.U.F., 1984, Pp. 187-213.

, (1932) Nouvelles conférences sur la psychanalyse, Paris, Gallimard, 1952.

, (1937) "Construcrions dans l'analyse ", Résultats, idées, problèmes II, 1921-1938, Paris, P.U.F., 1987, pp. 269-281.

HANLY, Charles, "On Being and Dreaming ", Psychoanalysis and Philosophy, Pp. $155-187$.

LAZEROWITZ, Morris, The Structure of Metaphysics, London, Routledge and Kegan Paul, 1955.

, "The Relevance of Psychoanalysis to Philosophy ", Psychoanalysis, Scientific Metbod and Philosophy, éd. par Sidney Hook, New York, New York University Press, 1959, pp. 133-156.

, Studies in Metaphilosophy, London, Routledge and Kegan Paul, 1964.

, Philosophy and Illusion, London, George Allan \& Unwin Ltd. et New York, Humanities Press, 1968.

, The Language of Philosophy. Freud and Wittgenstein, Dordrecht et Boston, D. Reidel Publishing Company, Boston Studies in the Philosophy of Science, Vol. LV, 1977. 
MOUNIIR, Emmanuel, Introduction aux existentialismes, Paris, Gallimard, 1962.

OSTOW, Murtimer et Ben-Ami SCHARFSTEIN, "The Need to Philosophize", Prychoanalysis and Philosophy, pp. 258-279.

SARTRE, Jean-Paul, L'êtré et le néant, Paris, Gallimard, 1984.

, Situations IV, Paris, Gallimard, 1964.

, Les mots, Paris, Gallimard, 1975.

SC.HARFSTEIN, Ben-Amiet Mortimer OSTOW, "The Need to Philosophize", Psychrounalysis and Philosophy, pp. 258-279.

SPINOZA, Baruch de, L'Éthique, Paris, Gallimard, 1964

STERN, Karl, Refus de la femme. Essai, Montréal, Éditions HMH, 1968.

VÉDRINE, Hélène, “Comment mettre Roquentin au travail... ou Bachelard et Sartre sur l’imaginaire ", Gaston Bachelard. Profils épistémologiques, éd., par Ciuy Lafrance, Ottawa, Presses de l'Université d'Ottawa, 1987. PP. $109-119$

WISDOM,J.O., "The Unconscious Origin of Schopenhauer's Philosophy », The Internutional Journal of Psycho-Analysis, 1945, Vol. XXVI, pp. 44-52. , "Three Dreams of Descartes», The International Journal of PsychoAnalysis, 1947, Vol. XXVIII, Pp. 11-18.

, The Unconfcious Origin of Berkeley's Philosophy, London, The Hogarth Press and the Institute of Psycho-Analysis, 1953.

, Philorophy and Its Place in Our Culture, New York, London, Paris, Gordon and Breach Science Publishers, 1975. 